\title{
Factor Analysis Based Selections
}

\author{
Sylvia Encheva ${ }^{1,2}$ \\ ${ }^{1}$ Stord/Haugesund University College,Bjørnsonsg. 45, \\ 5528 Haugesund, Norway \\ ${ }^{2}$ Uni Research Polytec AS, Sørhauggt. 128, \\ 5527 Haugesund, Norway
}

\begin{abstract}
Merger in higher education has been of scholarly interest to researchers in various fields. This work is devoted to challenges related to partner selection for an feasible merger. A systematic approach is proposed based on describing educational organizations via several predefined key numbers from the one hand and their expectations from the other hand. Methods from Boolean factor analysis, formal concept analysis, and Belnap's logic are further employed in an attempt of drawing meaningful conclusions.
\end{abstract}

Keywords-Boolean factor analysis; Formal concept analysis; Belnap's logic

\section{INTRODUCTION}

Merger in higher education has been seriously discussed for more than four decades, [6], [7]. Reasons, forms and outcomes of mergers are of continues interest to the research community. Interesting aspects of mergers of non-university colleges in Norway are well presented in [10]. Importance of geography and culture in mergers is emphasized in [11] while the authors of [5] pay special attention on challenges in merger processes.

In this work we consider problems related to selection of potential partners for an intended merger. In particular we are looking at university colleges and universities that have already expressed positive opinions about joining other higher education organizations. A merger in higher education is both time consuming and laborious. In such cases it is not a big surprise that a number of important questions arises and answers should involve several factors. One of these questions, all education organizations have to answer, is with whom they would like to merge. We propose a systematic approach for partners selection supported by Belnap's logic [1] and Boolean factor analysis, [2].

\section{PRELIMINARIES}

The semantic characterization of a four-valued logic for expressing practical deductive processes is presented in [1]. The idea in Belnap's approach is to develop a logic that is not that dependable of inconsistencies. The Belnap's logic has four truth values 'T, F, Both, None'. The meaning of these values can be described as follows: an atomic sentence is stated to be true only (T), an atomic sentence is stated to be false only $(\mathrm{F})$, an atomic sentence is stated to be both true and false, for instance, by different sources, or in different points of time (Both), and an atomic sentences status is unknown. That is, neither true, nor false (None).

Let $P$ be a non-empty ordered set. If $\sup \{x, y\}$ and inf $\{x, y\}$ exist for all $x, y \in P$, then $P$ is called a lattice [4]. In a lattice illustrating partial ordering of knowledge values, the logical conjunction is identified with the meet operation and the logical disjunction with the join operation.

A formal concept of $\langle X, Y, C\rangle$ is any pair $\langle E, F\rangle$ consisting of $E \subseteq X$ (so-called extent) and $F \subseteq Y$ (so-called intent) satisfying $E^{\uparrow}=F$ and $F^{\downarrow}=E$ where

$$
E^{\uparrow}=\{y \in Y \mid \text { for each } x \in X:\langle x, y\rangle \in C\},
$$

and

$$
F^{\downarrow}=\{x \in X \mid \text { for each } y \in Y:\langle x, y\rangle \in C\}
$$

When a set of objects is defined by a set of variables, it is useful to find a set of new variables (referred to as factors) describing the set of objects where the cardinality of the factor set is smaller than the cardinality of the variable set. Using notations as in [3], relationships among objects, variables and factors are shown in [2] where a matrix $I$ illustrates objects and variables dependencies, matrix $A$ illustrates objects and factors dependencies, and matrix $B$ illustrates factor and variables dependencies.

Assume two Boolean data tables $C_{1}$ and $C_{2}$, interconnected with an objects-attributes relation $\mathcal{R}_{C_{1} C_{2}}$, [9]. Relational factors based on $\mathcal{R}_{C_{1} C_{2}}$ can be found applying Definition 1 .

Definition 1: [9] Relation factor (pair factor) on data tables $C_{1}$ and $C_{2}$ is a pair $\left\langle F_{1}^{i}, F_{2}^{j}\right\rangle$, where $F_{1}^{i} \in \mathcal{F}_{1}$ and $F_{2}^{j} \in \mathcal{F}_{2}\left(\mathcal{F}_{i}\right.$ denotes set of factors of data table $C_{i}$ ) and satisfying relation $\mathcal{R}_{C_{1} C_{2}}$.

In order to select factors of practical importance we adopt the $\alpha$-approach as in [9]: for any $\alpha \in[0,1], F_{1}^{i}$ and $F_{2}^{j}$ form a pair factor $\left\langle F_{1}^{i}, F_{2}^{j}\right\rangle$ if the following holds:

$$
\frac{\left|\left(\bigcap_{k \in \operatorname{extent}\left(F_{1}^{i}\right)} \mathcal{R}_{k}\right) \cap \operatorname{intent}\left(F_{2}^{j}\right)\right|}{\left|\left(\bigcap_{k \in \operatorname{extent}\left(F_{1}^{i}\right)} \mathcal{R}_{k}\right)\right|} \geq \alpha .
$$

The problem has been previously addressed in [8].

\section{MERGERS}

All data in this article is taken from publicly available sources. Eleven higher educational institutions in Norway have been selected according to their interests in eventual merger. The names of these institutions are left anonymous since there are currently undergoing negotiations. Six university colleges and five universities are described by eight attributes. The attribute selection is based on the so called 'key numbers' (further on referred to as 'attributes') educational institutions 
use to present themselves. The numerical nature of such attributes allows application of mathematical methods in the process of drawing conclusions. Political reasonings however are beyond the scope of this work.

General attributes describing each educational institution and at the same time being of interest with respect to eventual merger are: academic degrees and titles of faculties, educational programs on graduate level, research articles published via predefined channels per faculty, and amount of obtained external funding. There is a national committee updating regularly a large list of national and international publication channels and classifying them as belonging to level one, level two or excluded from that list, where level two is the highest. Every educational institution receives certain financial support from the state corresponding to the amount of recognized by the state number of publications and the amount received from externally funded projects. the following attributes are further on divided into two parts: number of faculties with a $\mathrm{PhD}$ or equivalent $\left(A_{1}\right)$, number of faculties with professorship or equivalent $\left(A_{2}\right)$; number of master programs $\left(A_{3}\right)$, number of $\mathrm{PhD}$ programs $\left(A_{4}\right)$, amount of published research articles at level one per faculty $\left(A_{5}\right)$, amount of published research articles at level two per faculty $\left(A_{6}\right)$; amount of obtained external national funding $\left(A_{7}\right)$, amount of obtained external international funding $\left(A_{8}\right)$.

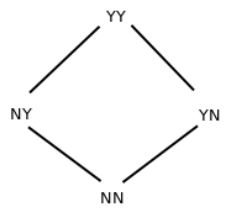

Fig. 1: Attributes' classification

These attributes are recognized by all interested parties but a closer look at Table I and Table II shows that they can be placed in four sets according to how an organization is presented and what that organization is expecting from a future partner: attributes that an organization possesses and is interested in a partner that also possesses them (YY), attributes that an organization does not possess but is interested in a partner that possesses them (NY), attributes that an organization possesses but is not necessarily that particular that a partner possesses them as well (YN), attributes that an organization does not possess and is also not necessarily that particular that a partner possesses them as well (NN). Based on this, attributes can be classified with the four valued Belnap's logic, Fig. 1. An attribute can be placed in any of the four sets in Fig. 1 depending on which organization is considering it. The majority of appearances can be used as an indication for the general importance of that attribute. Thus $f$. ex. if one attribute is classified four times as (YY) and two times as (YN) while another one is classified three times as (NY) and three times as $(\mathrm{NN})$, then the first one should have priority compare to the second. Such classification can assist in the process of organizational choices of criteria for partner selection.

The university colleges are denoted by $U C_{1}, U C_{2}, U C_{3}, U C_{4}, U C_{5}, U C_{6}$ while the universities are denoted by $U_{1}, U_{2}, U_{3}, U_{4}, U_{5}$.
TABLE I: University Colleges

\begin{tabular}{|c|c|c|c|c|c|c|c|c|}
\hline & $A_{1}$ & $A_{2}$ & $A_{3}$ & $A_{4}$ & $A_{5}$ & $A_{6}$ & $A_{7}$ & $A_{8}$ \\
\hline \hline$U C_{1}$ & $\times$ & & $\times$ & $\times$ & & $\times$ & & $\times$ \\
\hline$U C_{2}$ & $\times$ & $\times$ & & & $\times$ & & & \\
\hline$U C_{3}$ & & & $\times$ & & $\times$ & $\times$ & $\times$ & \\
\hline$U C_{4}$ & $\times$ & & $\times$ & & & $\times$ & & \\
\hline$U C_{5}$ & & $\times$ & & $\times$ & $\times$ & & & \\
\hline$U C_{6}$ & & $\times$ & $\times$ & & & $\times$ & $\times$ & $\times$ \\
\hline
\end{tabular}

\section{TABLE II: Universities}

\begin{tabular}{|c|c|c|c|c|c|c|c|c|}
\hline & $A_{1}$ & $A_{2}$ & $A_{3}$ & $A_{4}$ & $A_{5}$ & $A_{6}$ & $A_{7}$ & $A_{8}$ \\
\hline \hline$U_{1}$ & & $\times$ & $\times$ & & $\times$ & $\times$ & $\times$ & $\times$ \\
\hline$U_{2}$ & $\times$ & & & $\times$ & $\times$ & & $\times$ & $\times$ \\
\hline$U_{3}$ & & $\times$ & $\times$ & $\times$ & $\times$ & $\times$ & & \\
\hline$U_{4}$ & $\times$ & & $\times$ & & $\times$ & $\times$ & $\times$ & \\
\hline$U_{5}$ & & $\times$ & & $\times$ & & & & $\times$ \\
\hline
\end{tabular}

We are applying methods from Boolean factor analysis which in this work should be understood in the following way: an object possess an attribute if the value of that attribute is above the average (for university colleges and universities respectively), and similarly an object does not possess an attribute if the value of that attribute is below the average (again for university colleges and universities respectively). For factor selection we follow [3].

Factors obtained from educational organizations and their attributes are of interest to our investigations if they share at least two elements. When only two educational organizations are considering a merger they may apply a reasoning that goes beyond the scope of our current research. Negotiation processes involving more than two partners however require different reasoning than the one applied in the case of two partners. The process is both time consuming and laborious, therefore a systematic approach is needed for preselecting appropriate partners for detailed considerations.

Relation $\mathcal{R}_{C_{U C} C_{U}}$ between university colleges and attributes of universities is presented in Table III.

A list of selected factors obtained from university colleges and their attributes:

$$
\begin{aligned}
& F_{1}^{U C}-\left\langle\left\{U C_{1}, U C_{4}\right\},\left\{A_{1}, A_{3}, A_{6}, A_{8}\right\}\right\rangle, \\
& F_{2}^{U C}-\left\langle\left\{U C_{1}, U C_{5}\right\},\left\{A_{4}\right\}\right\rangle, \\
& F_{3}^{U C}-\left\langle\left\{U C_{2}, U C_{5}\right\},\left\{A_{2}, A_{5}\right\}\right\rangle, \\
& F_{4}^{U C}-\left\langle\left\{U C_{3}, U C_{6}\right\},\left\{A_{3}, A_{6}, A_{7}\right\}\right\rangle, \\
& F_{5}^{U C}-\left\langle\left\{U C_{1}, U C_{2}, U C_{4},\right\},\left\{A_{1}, A_{6}, A_{8}\right\}\right\rangle,
\end{aligned}
$$

TABLE III: Relation $\mathcal{R}_{C_{U C} C_{U}}$

\begin{tabular}{|c|c|c|c|c|c|c|c|c|}
\hline & $A_{1}$ & $A_{2}$ & $A_{3}$ & $A_{4}$ & $A_{5}$ & $A_{6}$ & $A_{7}$ & $A_{8}$ \\
\hline$U C_{1}$ & & $\times$ & & $\times$ & $\times$ & & $\times$ & $\times$ \\
\hline$U C_{2}$ & $\times$ & $\times$ & & & $\times$ & $\times$ & & \\
\hline$U C_{3}$ & $\times$ & & $\times$ & $\times$ & $\times$ & & $\times$ & \\
\hline$U C_{4}$ & & $\times$ & $\times$ & $\times$ & & $\times$ & $\times$ & $\times$ \\
\hline$U C_{5}$ & & $\times$ & & $\times$ & & $\times$ & $\times$ & \\
\hline$U C_{6}$ & $\times$ & & $\times$ & & $\times$ & $\times$ & & \\
\hline
\end{tabular}


TABLE IV: Relation Factors

\begin{tabular}{|c|c|c|c|c|c|c|c|c|c|c|c|c|}
\hline & $F_{1}^{U}$ & $F_{2}^{U}$ & $F_{3}^{U}$ & $F_{4}^{U}$ & $F_{5}^{U}$ & $F_{6}^{U}$ & $F_{7}^{U}$ & $F_{8}^{U}$ & $F_{9}^{U}$ & $F_{10}^{U}$ & $F_{11}^{U}$ & $F_{12}^{U}$ \\
\hline \hline$F_{1}^{U C}$ & & & & $\times$ & $\times$ & $\times$ & $\times$ & $\times$ & & & $\times$ & \\
\hline$F_{2}^{U C}$ & & $\times$ & $\times$ & & & & & $\times$ & & $\times$ & & \\
\hline$F_{3}^{U C}$ & $\times$ & $\times$ & & $\times$ & $\times$ & & $\times$ & & & & & $\times$ \\
\hline$F_{4}^{U C}$ & $\times$ & & & $\times$ & & $\times$ & $\times$ & & $\times$ & & $\times$ & \\
\hline$F_{5}^{U C}$ & & & & & $\times$ & & & & & & & \\
\hline$F_{6}^{U C}$ & & & & $\times$ & $\times$ & $\times$ & & $\times$ & & & & \\
\hline$F_{7}^{U C}$ & & $\times$ & $\times$ & & & & & $\times$ & & $\times$ & & \\
\hline$F_{8}^{U C}$ & & & & $\times$ & $\times$ & $\times$ & & $\times$ & & & $\times$ & \\
\hline
\end{tabular}

$$
\begin{aligned}
& F_{6}^{U C}-\left\langle\left\{U C_{1}, U C_{4}, U C_{6}\right\},\left\{A_{3}, A_{6}, A_{8}\right\}\right\rangle, \\
& F_{7}^{U C}-\left\langle\left\{U C_{2}, U C_{5}, U C_{6}\right\},\left\{A_{2}\right\}\right\rangle, \\
& F_{8}^{U C}-\left\langle\left\{U C_{1}, U C_{3}, U C_{4}, U C_{5}\right\},\left\{A_{3}, A_{6}\right\}\right\rangle .
\end{aligned}
$$

A list of selected factors obtained from universities and their attributes:

$$
\begin{aligned}
& F_{1}^{U}-\left\langle\left\{U_{1}, U_{2}\right\},\left\{A_{4}, A_{7}\right\}\right\rangle, \\
& F_{2}^{U}-\left\langle\left\{U_{1}, U_{3}\right\},\left\{A_{2}, A_{4}, A_{5}\right\}\right\rangle, \\
& F_{3}^{U}-\left\langle\left\{U_{1}, U_{5}\right\},\left\{A_{2}, A_{5}, A_{7}\right\}\right\rangle, \\
& F_{4}^{U}-\left\langle\left\{U_{2}, U_{3}\right\},\left\{A_{3}, A_{4}\right\}\right\rangle, \\
& F_{5}^{U}-\left\langle\left\{U_{2}, U_{4}\right\},\left\{A_{1}, A_{4}, A_{6}\right\}\right\rangle, \\
& F_{6}^{U}-\left\langle\left\{U_{2}, U_{5}\right\},\left\{A_{3}, A_{7}\right\}\right\rangle, \\
& F_{7}^{U}-\left\langle\left\{U_{3}, U_{4}\right\},\left\{A_{4}, A_{8}\right\}\right\rangle, \\
& F_{8}^{U}-\left\langle\left\{U_{3}, U_{5}\right\},\left\{A_{2}, A_{3}, A_{5}\right\}\right\rangle, \\
& F_{9}^{U}-\left\langle\left\{U_{1}, U_{2}, U_{5}\right\},\left\{A_{7}\right\}\right\rangle, \\
& F_{10}^{U}-\left\langle\left\{U_{1}, U_{3}, U_{5}\right\},\left\{A_{2}, A_{5}\right\}\right\rangle, \\
& F_{11}^{U}-\left\langle\left\{U_{2}, U_{3}, U_{5}\right\},\left\{A_{3}\right\}\right\rangle, \\
& F_{12}^{U}-\left\langle\left\{U_{1}, U_{2}, U_{3}, U_{4}\right\},\left\{A_{4}\right\}\right\rangle .
\end{aligned}
$$

Relational factors obtained via $\mathcal{R}_{C_{U C} C_{U}}$ are shown in Table IV.

Factors are represented applying the $\alpha$-approach.

Factor $\left\langle F_{1}^{U C}, F_{4}^{U}\right\rangle$ can be interpreted as - university colleges with number of master programs below the average are interested to merge with universities with number of master programs above the average. Note that there are other university colleges apart from the ones in $F_{1}^{U C}$ that express the same interest.

Factor $\left\langle F_{2}^{U C}, F_{7}^{U}\right\rangle$ represent another relation all university colleges with number of $\mathrm{PhD}$ programs above the average are interested to merge with universities with number of $\mathrm{PhD}$ programs above the average. Such conclusions can be also depicted from concepts generated with application of formal concept analysis [4] on Table IV.

Factor $\left\langle F_{8}^{U C}, F_{5}^{U}\right\rangle$ indicates that university colleges publishing research results at level two per faculty above the average are interested to merge with universities publishing research results at level two per faculty above the average as well.

Factor $\left\langle F_{3}^{U C}, F_{10}^{U}\right\rangle$ can be interpreted as - university colleges with a number faculties with professorship or equivalent and publishing research results at level one per faculty above the average are interested to merge with universities with number of faculties with professorship or equivalent and publishing research results at level one per faculty above the average.

The above extracted factor pairs illustrate that groups of educational organizations in the presented set share at least one relatively general interest, partner universities should have similar level of achievements within selected priority areas. At a glance these types of preferences seem to be quite natural. If however an organization has to show official interest in a merger with other organizations it is possible to use absence of expressed interest in some factors as an opportunity to release constrains and increase chances for successful negotiations.

Note that if we were to explore a couple of organizations considering eventual merger we would end up with somewhat different outcomes. This can be explained with specific priorities, local interests and nonetheless institutional culture which is however difficult to generalize.

In a future work we will investigate which $\alpha$ values appear to be sufficient for completed mergers.

\section{CONClusion}

Tendencies found in this article can be used in future planing and negotiations for merging organizations from higher educational sector. Thus a single institution can adjust its priority areas in order to meet expectations from other organizations and to make itself a more desirable partner.

Recommendations resulting from such research relate institutions' self-images, their views about other institutions, and their expectations from potential partners. Therefore conclusions coming from such analysis need to be taken as indications only and be further discussed by authorized government bodies.

\section{REFERENCES}

[1] N. J. Belnap, A useful four.valued logic, Modern uses of multiplevalued logic, J.M. Dunn and G. Epstain (eds), D. Reidel Publishing Co., Dordrecht, pp. 8-37, 1977.

[2] R. Belohlvek and V. Vychodil, V., Formal concepts as optimal factors in boolean factor analysis: Implications and experiments, In: Eklund, P.W., Diatta, J., Liquiere, M., (eds.) CLA. CEUR Workshop Proceedings, vol. 331. CEUR-WS.org, 2007.

[3] R. Belohlvek and V. Vychodil, V., Discovery of optimal factors in binary data via a novel method of matrix decomposition, J. Comput. Syst. Sci., vol. 76(1), pp. 3-20, 2010.

[4] B. Ganter and R. Wille, Formal Concept Analysis, Springer, 1999.

[5] S. R. Giessner, V. G. Tendayi, S. Otten, D. J. Terry, and S. Tuber, The challenge of merging: Merger patterns, pre-merger status and merger support, Personality and Social Psychology Bulletin, vol. 32 (3), pp. 339-352, 2006

[6] G. Harman, Restructuring higher education systems through institutional mergers: Australian experience, 19811983, vol. 15 (6), pp. 567-586, 1986.

[7] K. Harman and L. Meek, Merger revisited: international perspectives on mergers in higher education, Higher Education, vol. 44, pp.1-4, 2002.

[8] M. Huchard, A. Napoli, H. M. Rouane, and P. Valtchev, A proposal for combining formal concept analysis and description logics for mining relational data, ICFCA, 2007.

[9] M. Krmelova and M. Trnecka, Boolean Factor Analysis of MultiRelational Data, CLA 2013, Laboratory L3i, University of La Rochelle, pp. 187-198, 2013. 
[10] S. Kyvik, The merger of non-university colleges in Norway, Higher Education, vol. 44, pp. 53-72, 2002.

[11] J.D. Norgåd and O.J. Skodvin, The importance of geography and culture in mergers: A Norwegian institutional case study, Higher Education, vol. 44, pp. 73-90, 2002. 